\title{
PREDICTION OF INITIAL PRODUCT DISTRIBUTIONS FROM PYROLYSIS OF NORMAL PARAFFINIC HYDROCARBONS*
}

\author{
Mitsuo MURATA, Shozaburo SAITO, \\ Akira AMANO and Siro MAEDA \\ Department of Chemical Engineering, Tohoku University, \\ Sendai, Japan
}

\begin{abstract}
The extension of the free-radical chain mechanism to heavy paraffinic hydrocarbons has been studied for practical purposes. A generalization of the mechanism is attempted based on the Markov chain theory and presented in a form suitable to computer analysis. This generalization allows easier prediction of the distributions of initial pyrolytic products. Better agreement with experimental results is achieved by taking into account the equilibrium among all the possible isomers for higher homologues of hydrocarbon free radicals. However, the calculation of products is complicated by inclusion of radical isomerization which has not been ascertained experimentally. A new model is constructed herein by avoiding a direct approach to the problem of isomerization, while preserving its effect on the distribution of products. In this treatment, radical isomerization is replaced by a hypothetical process that accompanies the decrease of primary radicals and the increase of secondary radicals. The initial product distributions predicted by the model show good agreement with the observations for a series of normal paraffins from $n$-butane to $n$-hexadecane.
\end{abstract}

\section{Introduction}

A knowledge of the distribution of products resulting from the pyrolysis of hydrocarbons and their mixtures, and thus the initial distribution of these products, is indispensable to the design of pyrolysis facilities. The free-radical chain mechanism developed by Rice et al.11-15) has been known useful for predicting the initial distribution of products from paraffinic hydrocarbons. However, its complicated way of treating the isomerization of free radicals restricts its application to pure light hydrocarbons, and difficulties arise when one tries to apply this mechanism to heavy hydrocarbons. Therefore, a method is desirable that permits prediction of the initial distribution of products from pyrolysis of heavy, as well as light, paraffinic hydrocarbons.

From this point of view, a generalization of the RiceKossiakoff mechanism (hereafter abbreviated as the R-K mechanism) was attempted based on the Markov

* Received on July 7, 1972

Presented at the 5th Autumn Meeting of the Soc. of Chem.

Engrs., Japan, Osaka, Oct. 1971

厂980 仙台市荒巻字青葉

東北大学工学部化学工学科 斉藤正三郎 chain theory and presented in a form suitable to computer analysis. However, at present the isomerization of free radicals is uncertain since this process has not been ascertained experimentally. As a result, a new model for predicting initial product distributions was constructed by avoiding a direct approach to the problem of isomerization, yet preserving its effect on the distribution of products. In this model, isomerization via intra-hydrogen atom transfer was simulated by a hypothetical process in such a way that the initial product distribution could be correctly reproduced. This approach will be shown by a comparison with experiments on normal paraffinic hydrocarbons from $\mathrm{C}_{4}$ to $\mathrm{C}_{16}$.

\section{Generalization of the R-K Mechanism}

Rice and Herzfeld ${ }^{13)}$ developed a free-radical chain mechanism to explain the general features of paraffinic hydrocarbon decompositions. However, it was found that the amount of ethylene yield predicted by this mechanism was too large in comparison with experiments. To account for this discrepancy, Rice and Kossiakoff ${ }^{14)}$ assumed that prior to rupture, the free radicals of $\mathrm{C}_{6}$ or higher hydrocarbons isomerize by ring formation. The results from the R-K mechanism 
exhibit relatively good agreement with the experimental data on $n$-hexadecane and iso-dodecane by Voge et al. ${ }^{17)}$ and on $n$-heptane by Appleby et al. ${ }^{1)}$. In spite of that, the R-K mechanism has rarely been applied to heavy paraffinic hydrocarbons except in the above cases. This is because the allowable isomerization paths for free radicals become so complicated with increasing carbon number that the extension of this mechanism to heavier paraffinic hydrocarbons is practically intractable in its original form of treatment.

According to the R-K mechanism, the reaction path for $n$-decane is shown by Fig. 1, which involves radical isomerizations via 6- and 7-membered rings. A 1-decyl radical splits into one ethylene molecule and one 1octyl radical and the latter further isomerizes rapidly to 2-, 3-, or 4-form. Such a reaction path was simplified by combining formally two reaction steps, radical isomerization and radical decomposition, so that the reaction path could be expressed as a Markov chain as shown in Fig. 2. For example, part of this decomposition reaction may be quantitatively expressed as

$$
\begin{aligned}
& C_{10} \rightarrow \phi_{e}(10,1) 1-C_{10}+\phi_{e}(10,2) 2-C_{10}+\phi_{e}(10,3) 3-C_{10} \\
& +\phi_{e}(10,4) 4-C_{10}+\phi_{e}(10,5) 5-C_{10} \\
& \underline{1-C_{10}} \rightarrow \phi_{e}(8,1) \underline{1-C_{\dot{8}}}+\phi_{e}(8,2) \underline{2-C_{\dot{8}}}+\phi_{e}(8,3) \underline{3-C_{8}} \\
& +\phi_{e}(8,4) \underline{4-C_{8}}+C_{2} H_{4} \\
& 3-C_{8} \rightarrow \phi(8,3)\left(\underline{1-C_{4}}+\underline{C_{4} H_{8}}\right)+(1-\psi(8,3))\left(\underline{C_{1}}+\underline{C_{7} H_{14}}\right)
\end{aligned}
$$

where $\phi_{e}(n, m)$ denotes the normalized ratio of an $m$ $C_{n}$ radical to $C_{n}$ radicals in equilibrium, and $\phi(n, m)$ is the normalized ratio of an $m-C_{n}$ radical in rupture leading to a lighter olefinic compound when alternative bonds are available.

The underlined terms correspond to the states of the system for a Markov chain, and the coefficients to the one-step transition probabilities. Regarding those coefficients as forming elements of a matrix $\boldsymbol{P}$, we can calculate the probability of the system in a given state at $N$ step, from the following matrix multiplication:

$$
\boldsymbol{C}^{(N)}=\boldsymbol{C}^{(0)} \cdot \boldsymbol{P}^{N}
$$

where $\boldsymbol{C}^{(0)}$ and $\boldsymbol{C}^{(N)}$ denote the initial and the $N$-step probability vector, respectively. By using a computer, one can carry out this probability computation for relatively high molecular weight paraffinic hydrocarbons. The calculation made along this line for $n$ hexadecane by using the parameters, $\phi_{e}$ and $\phi$, given by Voge and Good, agrees very well with their results.

Recently the isotopic labeling experiments of McNesby et al..$^{5,6)}$ indicated the possibility of radical isomerization taking place via 5-, 6-, and 7-membered ring formation, which requires little strain energy. In particular, 6-membered ring formation requires zero strain energy, hence is expected to be the most important. However, there is at present no information as to the extent to which 5- and 7-membered rings contribute to the reaction as compared with 6-membered rings. Rice et al. and Voge et al. assumed a rapid isomerization equilibrium via 7- as well as 6-membered

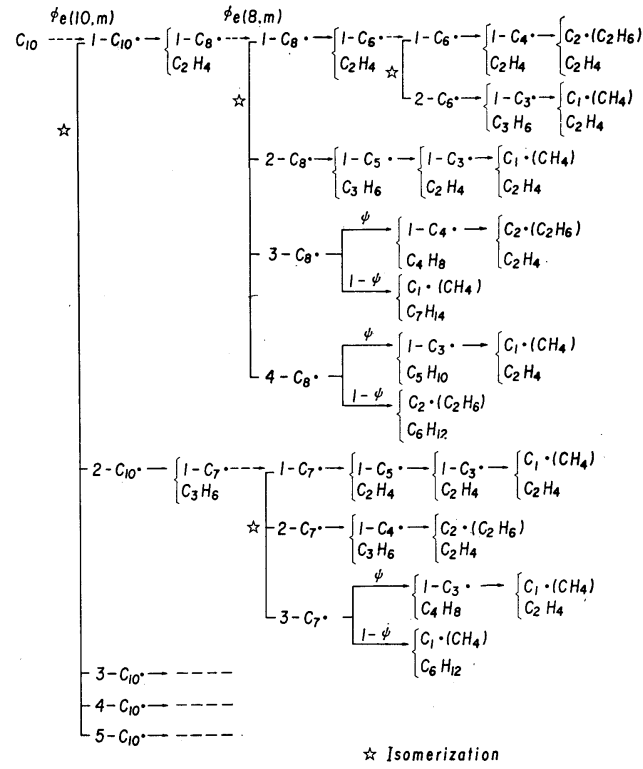

Fig. 1 Reaction path of $\boldsymbol{n}$-decane by the R-K mechanism

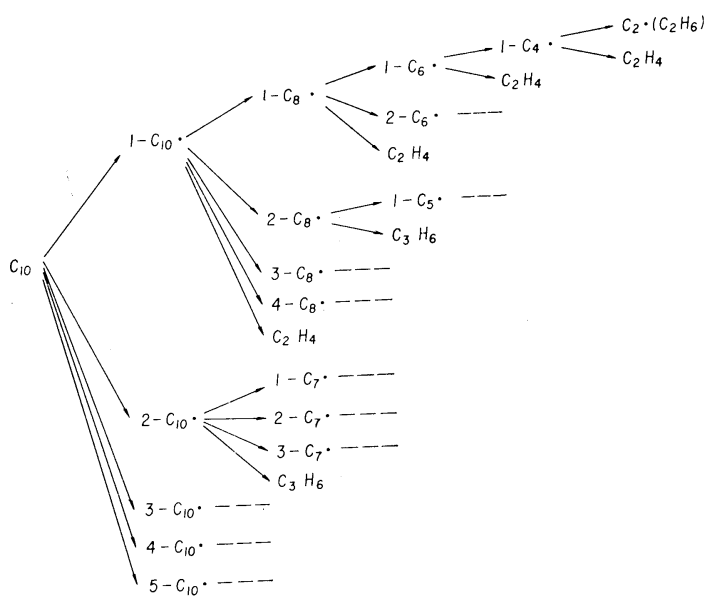

Fig. 2 Simplified reaction path of $\boldsymbol{n}$-decane

rings. Under this assumption, all the possible isomers are obtained by the transfer of a hydrogen atom for the radicals of $C_{8}$ or higher. The 5-membered ring process would not have been introduced since the same results are given by its addition. If the isomerization is limited to the process via 6 -membered rings, not all the possible isomers are obtained for the radicals of $C_{6}$ or higher. In the case of octyl radicals, for instance, the equilibrium is considered to be as follows:

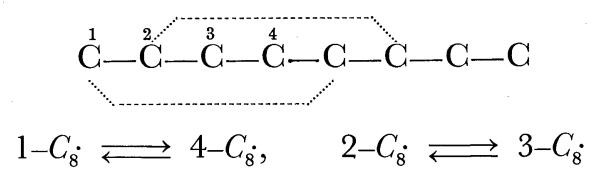

Therefore, a new radical produced by the transfer of a hydrogen atom from a 1-octyl radical is limited to the 4-form.

Prior to the calculation of the initial product distribution, it was investigated whether or not all the isomers for the radicals of $C_{8}$ or higher were produced by the transfer of a hydrogen atom. To do this, the 


\begin{tabular}{|c|c|c|c|}
\hline \multicolumn{4}{|c|}{ 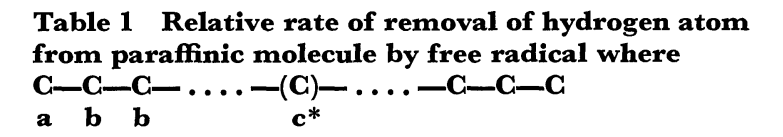 } \\
\hline $\begin{array}{l}\text { Position of } \\
\mathbf{H} \text { vacancy }\end{array}$ & $\begin{array}{l}\text { Statistical } \\
\text { factor } 11 \text { ) }\end{array}$ & $\begin{array}{c}\text { Relative } \\
\text { chance11) }\end{array}$ & $\begin{array}{l}\text { Relative } \\
\text { rate11) }\end{array}$ \\
\hline $\mathrm{a}$ & 6 & 1 & 6 \\
\hline b & 4 & $\exp (2000 / R T)$ & $4 \times \exp (2000 / R T)$ \\
\hline $\mathrm{c}^{*}$ & 2 & $\exp (2000 / R T)$ & $2 \times \exp (2000 / R T)$ \\
\hline
\end{tabular}

* These values should be used for a central carbon contained in a compound having odd number carbons.

values of the parameters, $\phi_{e}$ and $\phi$, are required. The values of $\phi_{e}$ were calculated from the normalized values of relative rates ${ }^{11)}, \phi$, shown in Table $\mathbf{1}$ by assuming that secondary radical was more stable than primary radical by $4 \mathrm{kcal}^{14)}$. The values of $\phi$ and $\phi$ were adopted from the work of Rice and Kossiakoff*14). The calculation of $\phi_{e}(n, m)$ is shown concretely for heptyl radicals in Appendix 1. Further, the following values were assigned to $\phi(n, m)$ :

$$
\phi(n, m)= \begin{cases}\frac{\exp (2000 / R T)}{1+\exp (2000 / R T)} & \text { for } m=3 \\ 0.5 & \text { for } m>3\end{cases}
$$

Two rupture processes were assumed to occur with equal likelihood, except when one of them yielded a methyl radical, which corresponded to $m=3$.

The calculation was performed for two cases: one involving isomerization via 6- and 7-membered rings and the other via 6-membered rings only. The results obtained for $n$-dodecane and $n$-hexadecane are compared with the corresponding experimental results in Figs. 3 and 4. Inclusion of 7-membered rings yields better agreement with experimental values than 6membered rings alone. The results indicate the necessity of taking into account isomerization via 7-membered rings and equilibrium among all the possible isomers for higher homologues of hydrocarbon free radicals.

\section{Equivalent Model for Predicting the Initial Product Distribution}

As mentioned previously, the extension of Eq.(2) to heavy paraffinic hydrocarbons is increasingly troublesome as the carbon number of radicals increases, because the many kinds of radical are produced. In view of the lack of information on radical isomerization mechanism, a simplified model for isomerization is presented here which is convenient and practical.

\section{1 Simplification of the R-K mechanism}

In this model, the radical isomerizations are represented by the recycling of primary radicals to the associated saturated hydrocarbons. The following assumptions will be made. Some of the primary radicals

\footnotetext{
* The values were re-examined on the data cited in the literature $3,8,9,10,16,18$ ) and were found reasonable.
}

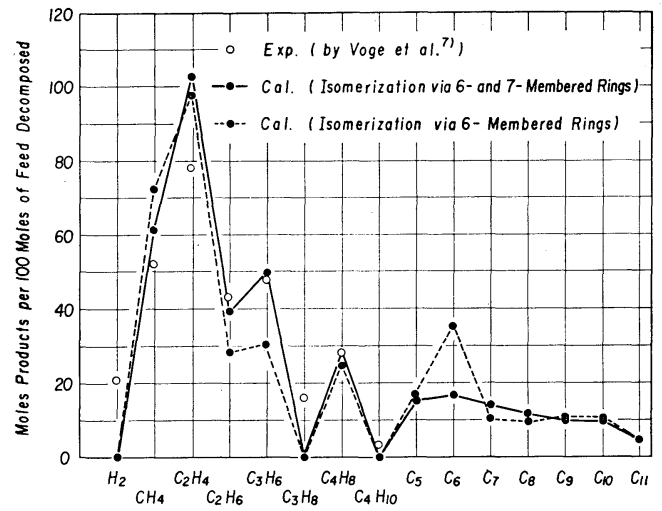

Fig. 3 Comparison of product distributions from $n$-dodecane with and without isomerization via 7 -membered rings at $550^{\circ} \mathrm{C}$ (Products of $C_{5}$ or higher denote olefinic compounds)

of $C_{3}$ or higher abstract hydrogen atoms intermolecularly, and then the resulting saturated hydrocarbons convert simultaneously to free radicals. Based on these assumptions, the decomposition reaction can be shown by the following schematic diagram:

$$
\begin{aligned}
& \frac{R \cdot}{\vdots}+\text { paraffin } \rightarrow \mathrm{RH}+\text { radical } \\
& \mathrm{RH}+\text { radical } \rightarrow \frac{R \cdot *}{\frac{R}{!}}+\text { paraffin }
\end{aligned}
$$

Here, if the radicals $R \cdot$ and $R \cdot *$ differ in the position of the $\mathrm{H}$ vacancy, they are regarded as corresponding to isomers. This translation may be called isomerization via an intermolecular hydrogen atom transfer reaction. The decomposition pattern of $n$-decane by this model, for example, is described as Fig. 5. Some 1-decyl radicals $1-C_{10}$ decompose by $\beta$-scission to give 1-octyl radicals $1-C_{8}$ and ethylene. The others give rise to $n$-decane by intermolecular hydrogen atom removal. The resulting $n$-decane then changes to free radicals $1-C_{10}$ to $5-C_{10}$. Similarly, $n$-octane and free radicals $2-C_{8}$ to $4-C_{\dot{8}}$ result from the decomposition of $1-C_{8}$. The isomerization in this case invariably accompanies the decrease of primary radicals and the increase of all possible secondary radicals. This is in accordance with the previous statement in section 1 that all the possible isomers must be taken into account.

To put the above concepts into practice, the decomposition reaction of, for example, $n$-decane shown in Fig. 5 was first expressed stoichiometrically. For the sake of simplicity, the reaction path and its stoichiometric equation of $n$-hexane are shown in Fig. 6 and Eq.(6), respectively.

$$
\begin{aligned}
\underline{C_{6} \rightarrow} & \phi(6,1)(1-\theta(6)) \underline{C_{6}}+\phi(6,1) \theta(6)(1-\theta(4)) \underline{C_{4}} \\
& +\phi(6,2)(1-\theta(3)) \underline{C_{3}}+\{\phi(6,1) \theta(6) \theta(4) \\
& +\phi(6,3) \phi(6,3)\} \underline{C_{2}}+\{\phi(6,2) \theta(3)+\phi(6,3) \\
& \times(1-\phi(6,3))\} \underline{C_{1}}+[\phi(6,1)\{\theta(6)+\theta(6) \theta(4)\} \\
& +\phi(6,2) \theta(3)] \underline{C_{2}}+\phi(6,2) \underline{C_{3}}=+\phi(6,3) \phi(6,3) \underline{C_{4}}= \\
& +\phi(6,3)(1-\phi(6,3)) \underline{C_{5}}=
\end{aligned}
$$




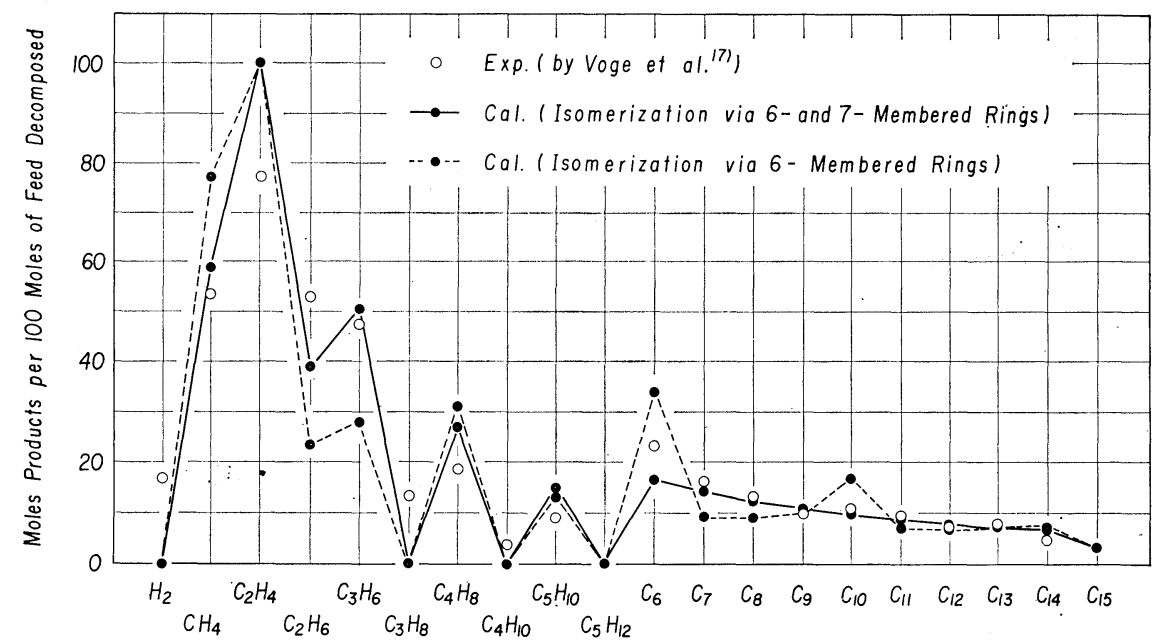

Fig. 4 Comparison of product distributions from $n$-hexadecane with and without isomerization via 7-membered rings at $500^{\circ} \mathrm{G}$ (Products of $C_{6}$ or higher denote olefinic compounds)

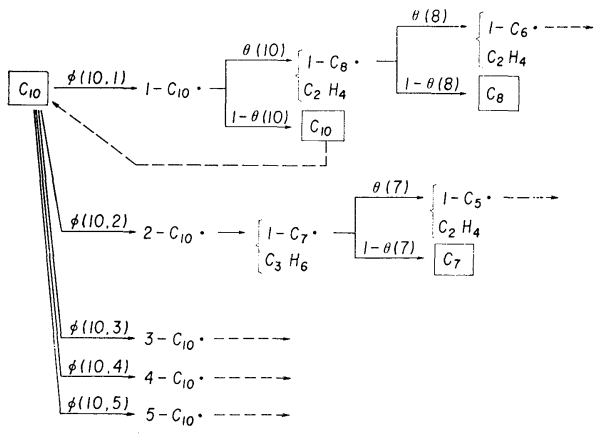

Fig. 5 Reaction path of $n$-decane by proposed model

Such stoichiometric equations are generalized for normal paraffinic hydrocarbons in Appendix 2. The initial product distributions are obtained through the following steps. In the first step, $n$-hexane decomposes into $n$-butane, propane, ethylene, etc., according to Eq.(6). In the second step, the resulting $n$-butane and propane decompose into lower hydrocarbons, obeying their respective decomposition scheme given by Appendix 2, and the $n$-hexane that has survived the first step resumes decomposition following Eq.(6). These steps are iterated until the saturated hydrocarbons other than ethane, such as propane, $n$-butane, and $n$-hexane, are consumed. This is illustrated schematically in Fig. 7, and is considered as a Markov chain. Therefore, the initial product distribution follows as a limit of $\boldsymbol{C}^{(N)}$ of Eq.(2).

A feature of the simplified model is that free radicals do not enter the equations explicitly, and the number of reactants to be treated in the calculation is drastically reduced. Application of Eq.(2) to heavier hydrocarbons is straightforward. Only the primary radicals in the previous assumptions were considered since the isomerization had influence mainly to the primary radicals. The assumptions are also supported by the fact that secondary radicals turn immediately into primary radicals by $\beta$-scission.

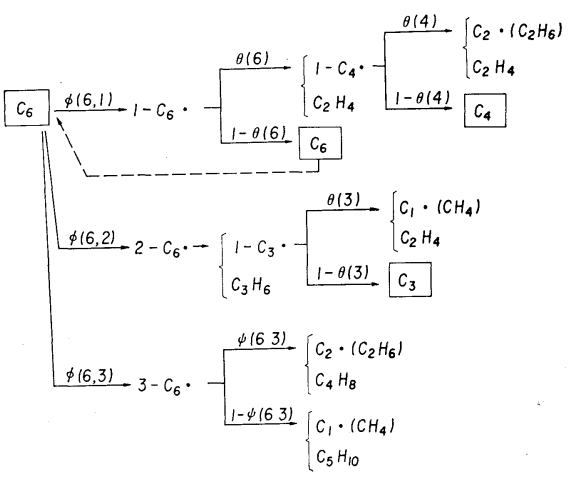

Fig. 6 Reaction path of $\boldsymbol{n}$-hexane by proposed model

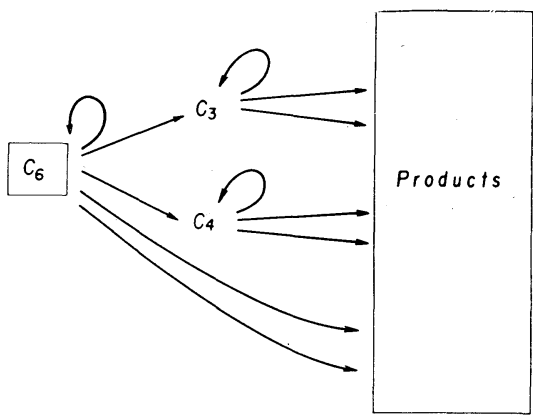

Fig. 7 Schematic representation of proposed model for $n$-hexane

As stated above, the present model was constructed by replacing the complicated isomerization processes of the R-K mechanism by a simpler hypothetical process. The advantage of this model consists in the fact that in evaluating the effect of radical isomerization on the initial distribution of pyrolytic products one requires only one parameter, $\theta(n)$, that represents the spectrum of $C_{n}$ radicals.

\section{2 Comparison of the proposed model with experiment}

The values given in section 1 for $\phi$ and $\phi$ were adopted for use here. The $\theta(n)$ were determined from 


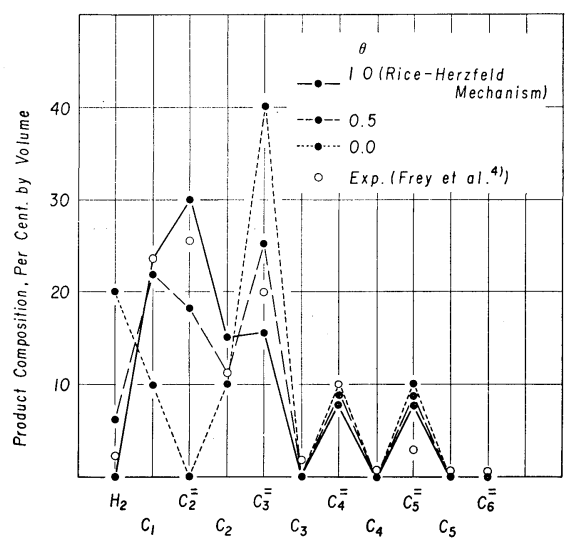

Fig. 8 Effect of $\boldsymbol{\theta}$ on product distribution from $n$-hexane at $\phi=0.5$ and $575^{\circ} \mathrm{C}$

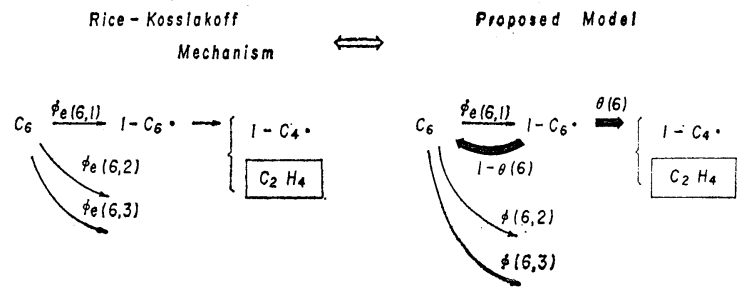

Fig. 10 Comparison of proposed model with the R-K mechanism for $n$-hexane

the experiments on $n-C_{4}$ to $n-C_{16}$ cited in the literature ${ }^{1,2,4,7-10,16-18)}$. The effect of $\theta(n)$ on the initial distribution is shown in Fig. 8 for $n$-hexane. It may be seen therefrom that the distribution is highly sensitive to the choice of $\theta(n)$. When $\theta(n)=1.0$, the model reduces to the Rice-Herzfeld mechanism. The figure suggests that the most probable values of $\theta(n)$ lie around 0.8 , independent of $n$. The distributions thus obtained agree with the experiments on $n$-butane to $n$-heptane. The distribution for $n$-hexane is typically represented in Fig. 9. With $n$-hexadecane, however, a large discrepancy was found in ethylene yield. Indications are that this is primarily due to the crude approximation for $\theta(n)$, which required re-examination, particularly for $n \geqslant 6$. According to the $\mathrm{R}-\mathrm{K}$ mechanism, the production ratios of radicals, $\phi$ and $\phi_{e}$ from $n$-hexane decomposing at $570^{\circ} \mathrm{C}$ are given respectively as

$$
\begin{aligned}
\phi(6,1) & =0.186, \quad \phi(6,2)=\phi(6,3)=0.407 \\
\phi_{e}(6,1) & =0.072, \quad \phi_{e}(6,2)=0.521, \quad \phi_{e}(6,3)=0.407
\end{aligned}
$$

The variation between $\phi$ and $\phi_{e}$ is exceptionally large with the primary radicals. Hence the $\theta(n)$ were adjusted so as to give the same ethylene yield from the primary radicals as in the $\mathrm{R}-\mathrm{K}$ mechanism. It then follows from Fig. 10 that

$$
\begin{aligned}
\phi_{e}(6,1)= & \phi(6,1) \cdot \theta(6)+\phi(6,1) \cdot \theta(6)\{\phi(6,1) \cdot(1-\theta(6))\} \\
& +\phi(6,1) \cdot \theta(6)\{\phi(6,1) \cdot(1-\theta(6))\}^{2} \\
& +\phi(6,1) \cdot \theta(6)\{\phi(6,1) \cdot(1-\theta(6))\}^{3}+\ldots \\
= & \frac{\phi(6,1) \cdot \theta(6)}{1-\phi(6,1)(1-\theta(6))}
\end{aligned}
$$

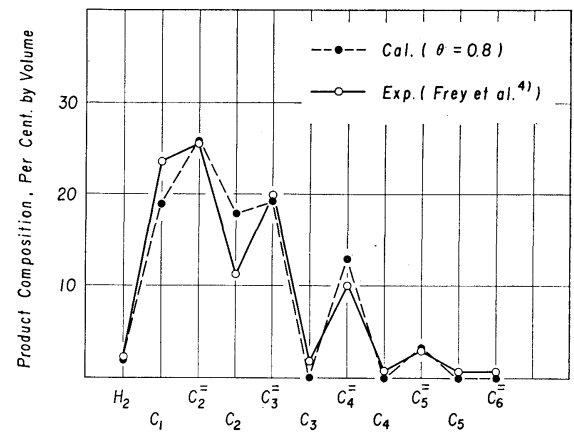

Fig. 9 Comparison of predicted products with experiment for $n$-hexane at $\theta=0.8$ and $575^{\circ} \mathrm{C}$

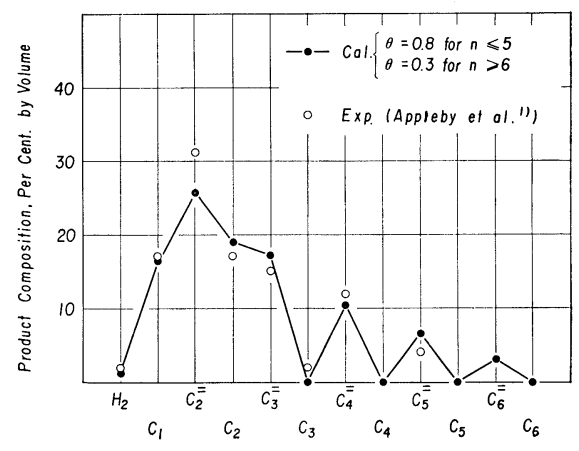

Fig. 11 Comparison of predicted products by proposed model with experiment for $n$-heptane at $580{ }^{\circ} \mathrm{C}$

By rearranging Eq.(8), one has

$$
\theta(6)=\frac{\phi_{e}(6,1)\{1-\phi(6,1)\}}{\phi(6,1)\left\{1-\phi_{e}(6,1)\right\}}
$$

The values thus obtained for $n$-heptane $\theta(7)$ and $n$ octane $\theta(8)$ were nearly equal to that of $n$-hexane $\theta(6)$. These three were also approximately independent of temperature in the range $500-700^{\circ} \mathrm{C}$. The $\theta(n)$ which gave the best fitting to the experimental results were finally determined as

$$
\theta(n)=\left\{\begin{array}{lll}
0.8 & \text { for } & n \leqslant 5 \\
0.3 & \text { for } & n \geqslant 6
\end{array}\right.
$$

By using these parameters, the initial product distributions of $n-C_{4}$ to $n$ - $C_{16}$ decomposition were recalculated. Some typical results are presented in Figs. 11 and 12, in good agreement with the experiments. The result predicted by the $\mathrm{R}-\mathrm{K}$ mechanism for $n$-hexadecane is also shown in Fig. 12 for the purpose of comparison.

Further, the effect of carbon number was investigated and the result is shown in Fig. 13. This effect is appreciable in hydrocarbons with $C$ up to 8 . Though not shown here, $C_{6}=C_{7}=C_{9}, C_{10}, C_{12}^{=}, C_{14}^{=}, C_{15}^{=}$exhibited the same trend as $C_{8}=$ and $C_{11}$.

Although the discussion has been limited to the case of pure components, the proposed method can be applied to mixtures of normal paraffinic hydrocarbons, provided the reactivity of each component is not affected by the existence of other components. However, few studies of the pyrolyses of mixtures are available, 


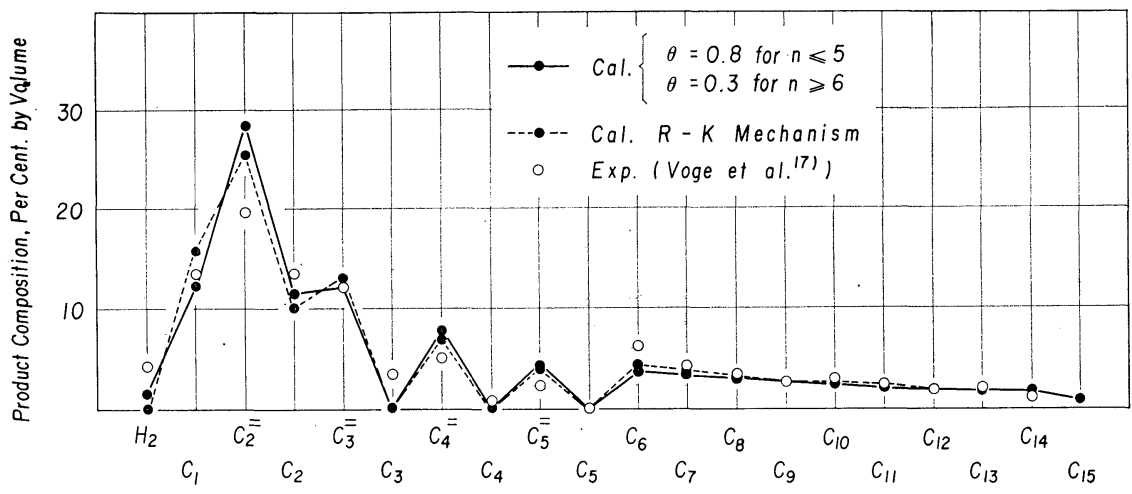

Fig. 12 Comparison of predicted products by proposed model with experiment for $\boldsymbol{n}$-hexadecane at $500^{\circ} \mathrm{C}$ (Products of $C_{6}$ or higher denote olefinic compounds)

and the effect of mixing remains to be ascertained.

\section{Conclusion}

A generalization of the $\mathrm{R}-\mathrm{K}$ mechanism was made based on the Markov chain theory. This allows computer analysis of pyrolytic products so that prediction becomes easier. The results indicate the necessity of taking into account all the possible isomers for higher homologues of hydrocarbon free radicals. Further, a new model is proposed to avoid the complexity of the reaction path arising from radical isomerizations. The initial products predicted by the model showed good agreement with the observations for $n-C_{4}$ to $n-C_{16}$.

\section{Appendix 1}

Calculation of $\phi_{e}(n, m)$ for heptyl radicals:

The isomerization via 6 - and 7-membered rings can be expressed as follows:

$$
\begin{array}{ll}
1-C_{7} \stackrel{K_{1}}{\rightleftharpoons} 2-C_{7} \\
& 1-C_{7} \stackrel{K_{2}}{\rightleftharpoons} 3-C_{7}
\end{array}
$$

where $K_{1}$ and $K_{2}$ denote equilibrium constants given by

$$
K_{1}=K_{2}=\exp (4000 / R T) \times \frac{4}{6}
$$

Thus the values of $\phi_{e}(7, m)$ are calculated as

$$
\begin{aligned}
& \phi_{e}(7,1)=(1-\phi(7,4)) \times \frac{1}{1+K_{1}+K_{2}} \\
& \phi_{e}(7,2)=(1-\phi(7,4)) \times \frac{K_{1}}{1+K_{1}+K_{2}} \\
& \phi_{e}(7,3)=(1-\phi(7,4)) \times \frac{K_{2}}{1+K_{1}+K_{2}}=\phi_{e}(7,2) \\
& \phi_{e}(7,4)=\phi(7,4)
\end{aligned}
$$

Similarly, isomerization via 6 -membered rings alone is

$$
\stackrel{1}{\mathrm{C}}-\stackrel{2}{\mathrm{C}}-\stackrel{3}{\mathrm{C}}-\stackrel{4}{\mathrm{C}}-\mathrm{C}-\mathrm{C}-\mathrm{G} \quad 1-C_{7} \stackrel{{ }^{2}}{\rightleftharpoons} 3-C_{\dot{7}}
$$

and

$$
\begin{aligned}
& \phi_{e}(7,1)=(1-\phi(7,2)-\phi(7,4)) \times \frac{1}{1+K_{2}} \\
& \phi_{e}(7,2)=\phi(7,2) \\
& \phi_{e}(7,3)=(1-\phi(7,2)-\phi(7,4)) \times \frac{K_{2}}{1+K_{2}}
\end{aligned}
$$

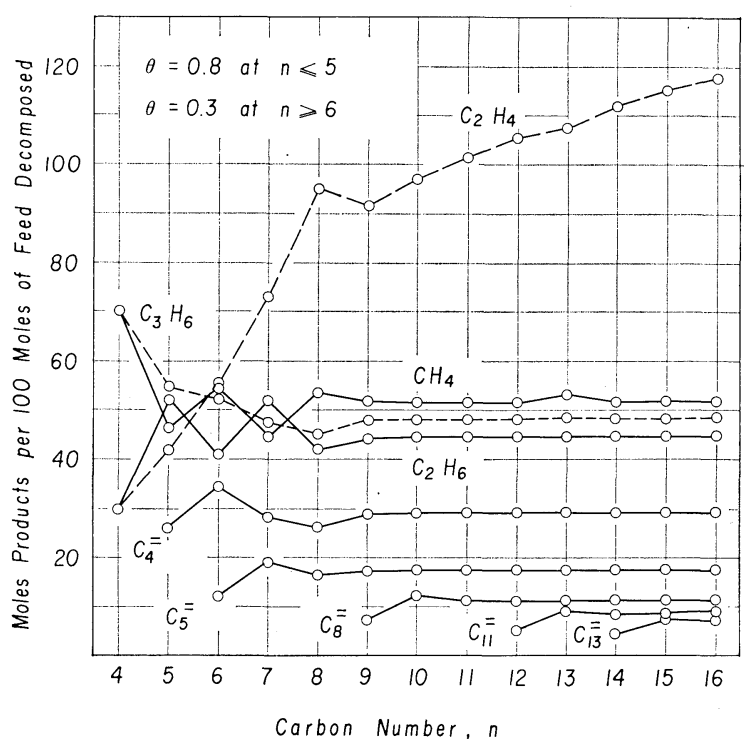

Fig. 13 Effect of carbon number on product distributions by proposed model at $700^{\circ} \mathrm{C}$

$$
\phi_{e}(7,4)=\phi(7,4)
$$

\section{Appendix 2}

Generalized stoichiometric equation based on the proposed model: For even numbers of $n(=2 p)$ :

$$
\begin{aligned}
\underline{C_{n}} & \rightarrow \phi(n, 1) \times\left[\left\{\sum_{j=1}^{p-1}\left(\prod_{k=j+1}^{p} \theta(2 k)\right)\right\} \underline{C_{2}}+(1-\theta(n)) \underline{C_{n}}\right. \\
& \left.+\sum_{j=2}^{p-1}\left\{\left(\prod_{k=j+1}^{p} \theta(2 k)\right) \times(1-\theta(2 j))\right\} \underline{C_{2 j}}+\left(\prod_{k=2}^{p} \theta(2 k)\right) \underline{C_{2}}\right]+\phi(n, 2) \\
& \times\left[\underline{C_{3}}=\left\{\sum_{j=1}^{p-2}\left(\prod_{k=j}^{p-2} \theta(2 k+1)\right)\right\} \underline{C_{2}}=(1-\theta(n-3)) \underline{C_{n-3}}\right. \\
& +\sum_{j=1}^{p-3}\left\{\left(\prod_{k=j+1}^{p-2} \theta(2 k+1)\right) \times(1-\theta(2 j+1))\right\} \underline{C_{2 j+1}} \\
& \left.+\left(\prod_{k=1}^{p-2} \theta(2 k+1)\right) \underline{C_{1}}\right]+\sum_{q=3}^{p} \mathbf{【} \phi(n, q) \times
\end{aligned}
$$

for even numbers of $q$ :

$$
\begin{aligned}
& {\left[\psi(n, q) \times \llbracket \underline{G_{q+1}=}+\left\{\sum_{j=1}^{(n-q-2) / 2}\left(\prod_{k=j}^{(n-q-2) / 2} \theta(2 k+1)\right)\right\} \underline{C_{2}}=\right.} \\
& +(1-\theta(n-q-1)) \underline{C_{n-q-1}}+{ }_{j=1}^{(n-q-4) / 2}\left\{\left(\prod_{k=j+1}^{(n-q-2) / 2} \theta(2 k+1)\right)\right. \\
& \left.\times(1-\theta(2 j+1))\} \underline{C_{2 j_{+1}}}+\left(\prod_{k=1}^{(n-q-2) / 2} \theta(2 k+1)\right) \underline{C_{1}}\right]
\end{aligned}
$$




$$
\begin{aligned}
& +(1-\psi(n, q)) \times \underline{\llbracket C_{n-q+2}}+\left\{\sum_{j=1}^{(q-4) / 2}\left(\prod_{k=j+1}^{(q-2) / 2} \theta(2 k)\right)\right\} \underline{C_{2}=} \\
& +(1-\theta(q-2)) \underline{C_{q-2}}+\sum_{j=2}^{(q-4) / 2}\left\{\left(\prod_{k=j+1}^{(q-2) / 2} \theta(2 k)\right)\right. \\
& \left.\times(1-\theta(2 j))\} \underline{C_{2 j}}+\left(^{(q-2) / 2} \prod_{k=2} \theta(2 k)\right) \underline{C_{2}} \rrbracket\right] 】
\end{aligned}
$$

for odd numbers of $q$ :

$$
\begin{aligned}
& {\left[\psi(n, q) \times \llbracket \underline{C_{q+1}}+\left\{\sum_{j=1}^{(n-q-3) / 2}\left(\prod_{k=j+1}^{(n-q-1) / 2} \theta(2 k)\right)\right\} \underline{C_{2}}=\right.} \\
& +(1-\theta(n-q-1)) \underline{C_{n-q-1}}+\sum_{j=2}^{(n-q-3) / 2}\left\{\left(\begin{array}{l}
(n-q-1) / 2 \\
\prod_{k=j+1}
\end{array} \theta(2 k)\right)\right. \\
& \times(1-\theta(2 j))\} \underline{C_{2 j}}+\left(\prod_{k=2}^{(n-q-1) / 2} \theta(2 k)\right) \underline{C_{2}} \rrbracket+(1-\psi(n, q)) \\
& \times \llbracket \underline{C_{n-q+2}}+\left\{\sum_{j=1}^{(q-3) / 2}\left(\prod_{k=j}^{(q-3) / 2} \theta(2 k+1)\right)\right\} \underline{C_{2}}= \\
& +(1-\theta(q-2)) \underline{C_{q-2}}+\sum_{j=1}^{(q-5) / 2}\left\{\left(\prod_{k=j+1}^{(q-3) / 2} \theta(2 k+1)\right)\right. \\
& \times(1-\theta(2 j+1))\} \underline{C_{2 j+1}}+\left(\prod_{k=1}^{(q-3) / 2} \theta(2 k+1)\right) \underline{\left.C_{1} \rrbracket\right] 】}
\end{aligned}
$$

For odd numbers of $n(=2 p-1)$ :

$$
\begin{aligned}
& \underline{C_{n}} \rightarrow \phi(n, 1) \times\left[\left\{\sum_{j=1}^{p-1}\left(\prod_{k=j}^{p-1} \theta(2 k+1)\right)\right\} \underline{C_{2}}=(1-\theta(n)) \underline{C_{n}}\right. \\
& +\sum_{j=1}^{p-2}\left\{\left(\prod_{k=j+1}^{p-1} \theta(2 k+1)\right) \times(1-\theta(2 j+1))\right\} \underline{C_{2 j+1}} \\
& \left.+\left(\prod_{k=1}^{p-1} \theta(2 k+1)\right) \underline{C_{1}}\right]+\phi(n, 2) \times\left[\underline{C_{3}}=\left\{\left[\sum_{j=1}^{p-3}\left(\prod_{k=j+1}^{p-2} \theta(2 k)\right)\right\} \underline{C_{2}}=\right.\right. \\
& +(1-\theta(n-3)) \underline{C_{n_{-3}}}+\sum_{j=2}^{p-3}\left\{\left(\prod_{k=j+1}^{p-2} \theta(2 k)\right) \times(1-\theta(2 j))\right\} \underline{C_{2 j}} \\
& \left.+\left(\prod_{k=2}^{p-2} \theta(2 k)\right) \underline{C_{2}}\right]+\sum_{q=3}^{p-1} \mathbf{I} \phi(n, q) \times \\
& \text { for even numbers of } q \text { : }
\end{aligned}
$$

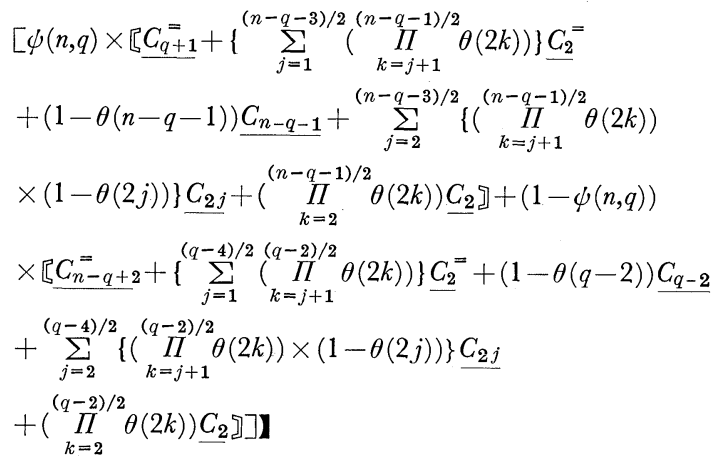

for odd numbers of $q$ :

$$
\begin{aligned}
& {\left[\psi(n, q) \llbracket \underline{C_{q+1}}+\left\{\sum_{j=1}^{(n-q-2) / 2}\left(\prod_{k=j}^{(n-q-2) / 2} \theta(2 k+1)\right)\right\} \underline{C_{2}=}\right.} \\
& +(1-\theta(n-q-1)) \underline{C_{n-q-1}}+\sum_{j=1}^{(n-q-4) / 2}\left\{\left(\prod_{k=j+1}^{(n-q-2) / 2} \theta(2 k+1)\right)\right. \\
& \times(1-\theta(2 j+1))\} \underline{C_{2 j_{+1}}}+\left(\prod_{k=1}^{(n-q-2) / 2} \theta(2 k+1) \underline{C_{1}}\right. \text { ] } \\
& +(1-\psi(n, q)) \times \llbracket \underline{G_{n-q+2}^{=}}+\left\{\sum_{j=1}^{(q-3) / 2}\left(\prod_{k=j}^{(q-3) / 2} \theta(2 k+1)\right)\right\} \underline{C_{2}}= \\
& +(1-\theta(q-2)) \underline{C_{q-2}}+\sum_{j=1}^{(q-5) / 2}\left\{\left(\prod_{k=j+1}^{(q-3) / 2} \theta(2 k+1)\right)\right. \\
& \left.\left.\times(1-\theta(2 j+1))\} \underline{C_{2 j_{+1}}}+\left(\prod_{k=1}^{(q-3) / 2} \theta(2 k+1)\right) \underline{C_{1}}\right]\right]
\end{aligned}
$$$$
+\phi(n, p) \times
$$

$$
\begin{aligned}
& {\left[\underline{C_{p+1}}+\left\{\sum_{j=1}^{(p-4) / 2}\left(\sum_{k=j+1}^{(p-2) / 2} \theta(2 k)\right)\right\} \underline{C_{2}}=\left(\sum_{k=2}^{(p-2) / 2} \theta(2 k)\right) \underline{C_{2}}\right.} \\
& +(1-\theta(p-2)) \underline{C_{p_{-2}}}+\sum_{j=2}^{(p-4) / 2}\left\{\left(\prod_{k=j+1}^{(p-2) / 2} \theta(2 k)\right)\right.
\end{aligned}
$$

$$
\left.\times(1-\theta(2 j))\} \underline{C_{2 j}}\right]
$$

for odd numbers of $p$ :

$$
\begin{aligned}
& {\left[\underline{C_{p+1}^{=}}+\left\{\sum_{j=1}^{(p-3) / 2}\left(\prod_{k=j}^{(p-3) / 2} \theta(2 k+1)\right)\right\} \underline{C_{2}}=\right.} \\
& +\left(\prod_{k=1}^{(p-3) / 2} \theta(2 k+1)\right) \underline{C_{1}}+(1-6(p-2)) \underline{C_{p-2}} \\
& \left.+\sum_{j=1}^{(p-5) / 2}\left\{\left(\prod_{k=j+1}^{(p-3) / 2} \theta(2 k+1)\right) \times(1-\theta(2 j+1))\right\} \underline{C_{2 j+1}}\right]
\end{aligned}
$$

in which expressions such as $\sum_{k=2}^{1}, \prod_{k=2}^{1}$, etc. that do not make sense mathematically are to be excluded and the order of the signs of grouping is $\mathbf{I}[\{\{(\quad)\}]] \mathbf{I}$.

\section{Acknowledgement}

Numerical calculation was carried out on the high-speed digital computer NEAC 2200 of Tohoku University.

\section{Nomenclature}

$$
\begin{aligned}
n & =\text { carbon number } \\
C_{n}= & \text { paraffinic hydrocarbon having } n \text { carbon atoms } \\
C_{n}^{=}= & \text {olefinic hydrocarbon having } n \text { carbon atoms } \\
m-C_{n} \cdot= & \text { free radical having } n \text { carbon atoms and having } \\
& \text { H-vacancy site at } m \text {-th carbon from the end of } \\
& \text { carbonskeleton } \\
K_{i}= & \text { equilibrium constant for radical isomerization } \\
\boldsymbol{C}^{(0)}= & \text { initial probability vector of feed } \\
\boldsymbol{C}^{(N)}= & N \text {-step probability vector } \\
\boldsymbol{P}= & \text { one-step transition probability matrix } \\
\phi(n, m)= & \text { normalized ratio of an } m \text { - } C_{n} \cdot \text { radical to } C_{n} \cdot \\
& \text { radicals calculated from relative rate of } \\
& \text { hydrogen-atom removal } \\
\phi_{e}(n, m)= & \text { normalized ratio of an } m-C_{n} \cdot \text { radical to } C_{n} \cdot \\
& \text { radicals in equilibrium via isomerization } \\
\phi(n, m)= & \text { normalized ratio of an } m-C_{n} \cdot \text { radical in rupture } \\
& \text { leading to a smaller olefinic compound when } \\
& \text { alternative bonds are available } \\
\theta(-] & \text { normalized ratio of primary } C_{n} \cdot \text { radicals } \\
& \text { decomposed }
\end{aligned}
$$

\section{Literature Gited}

1) Appleby, W. G., W. H. Avery and W. K. Meerbott: J. Am. Chem. Soc., 69, 2279 (1947)

2) Archibald, R. G., N. G. May and B. S. Greensfelder: Ind. Eng. Chem., 44, 1811 (1952)

3) Buekens, A. G. and G. F. Froment: $I \&$ EC, Process Design and Development, 7, 435 (1968)

4) Frey, F. E. and H. J. Hepp: Ind. Eng. Chem., 25, 441 (1933)

5) Gordon, A. S. and J. R. McNesby: J. Chem. Phys., 31, 853 (1959)

6) Gordon, A. S. and J. R. McNesby: ibid., 33, 1882 (1960)

7) Greensfelder, B. S. and H. H. Voge: Ind. Eng. Chem., 37, 514 (1945)

8) Marschner, R. F.: Ind. Eng. Chem., 30, 554 (1938)

9) Morgan, J. J. and J. C. Munday: Ind. Eng. Chem., 27, 1082 (1935)

10) Purnell, J. H. and C. P. Quinn: Proc. Roy. Soc. London, A270, $267(1962)$

1 ) Rice, F. O.: J. Am. Chem. Soc., 53, 1959 (1931)

12) Rice, F. O.: ibid., 55, 3035 (1933)

13) Rice, F. O. and K. F. Herzfeld: $i b i d ., 56,284$ (1934)

14) Rice, F. O. and A. Kossiakoff: ibid., 65, 590 (1943)

15) Rice, F. O. and K. K. Rice: "The Aliphatic Free Radicals", Baltimore, Johns Hopkins Press (1935)

16) Sagert, N. H. and K. J. Laidler: Can. J. Chem., 41, 838 (1963)

17) Voge, H. H. and G. M. Good: J. Am. Chem. Soc., 71, 593 (1949)

18) Wang, Y. L., R. G. Rinker and W. H. Corcoran: $I \& E C$, Fundamentals, 2, 161 (1963) 